\title{
The XMM Cluster Survey: Present status and latest results
}

\author{
P.T.P. Viana ${ }^{1,2, \star}$, N. Mehrtens ${ }^{3,4}$, C.D. Harrison ${ }^{5}$, A.K. Romer ${ }^{3,6}$, C.A. Collins ${ }^{7}$, M. Hilton ${ }^{8,9}$, B.

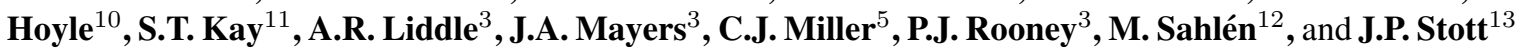 \\ ${ }^{1}$ Centro de Astrofísica, Universidade do Porto, Rua das Estrelas, 4150-762 Porto, Portugal \\ 2 Departamento de Física e Astronomia, Faculdade de Ciências, Universidade do Porto, Rua do Campo Alegre, 687, \\ 4169-007 Porto, Portugal \\ 3 Astronomy Centre, University of Sussex, Falmer, Brighton BN1 9QH, UK \\ 4 Department of Physics and Astronomy, Texas A\&M University, College Station, TX 77845-4242, USA \\ 5 Astronomy Department, University of Michigan, Ann Arbor, MI 48109, USA \\ ${ }^{6}$ SEPnet, South East Physics Network (www.sepnet.ac.uk), UK \\ 7 Astrophysics Research Institute, Liverpool John Moores University, Twelve Quays House, Egerton Wharf, Birkenhead \\ CH41 1LD, UK \\ 8 Centre for Astronomy \& Particle Theory, School of Physics \& Astronomy, University of Nottingham, Nottingham, \\ NG7 2RD, UK \\ 9 Astrophysics \& Cosmology Research Unit, School of Mathematics, Statistics \& Computer Science, University of \\ KwaZulu-Natal, Private Bag X54001, Durban, 4000, South Africa \\ 10 Institut de Ciéncies del Cosmos (ICCUB-IEEC), Departmento de Física, Martí i Franqués 1, 08034 Barcelona, Spain \\ 11 Jodrell Bank Centre for Astrophysics, School of Physics and Astronomy, The University of Manchester, Manchester, \\ M13 9PL, UK \\ 12 The Oskar Klein Centre for Cosmoparticle Physics, Department of Physics, Stockholm University, SE-106 91 Stock- \\ holm, Sweden \\ 13 Extragalactic and Cosmology Group, Department of Physics, University of Durham, South Road, Durham DH1 3LE, \\ UK
}

Received 2012 Aug 24, accepted 2012 Sep 20

Published online 2013 May 2

Key words galaxies: clusters: general - X-rays: galaxies: clusters - surveys

The XMM Cluster Survey (XCS) is a serendipitous search for galaxy clusters using all publicly available data in the XMM-Newton Science Archive. Our recent first data release (XCS-DR1) contains 503 optically confirmed groups and clusters, among which 256 new to the literature and 357 whose X-ray emission was detected for the first time. We discuss their properties and provide an update on the work being done. As examples of the applications of XCS-DR1, we mention the 17 fossil groups/clusters identified with the help of the Sloan Digital Sky Survey. Their brightest galaxies have stellar populations and star-formation histories which are similar to normal brightest cluster galaxies, but their stellar masses are significantly larger and correspond to a much bigger fraction of the total group/cluster optical luminosity. We also highlight the 15 clusters expected to be also detected by the Planck satellite, and characterize the expected overlap between the final $\mathrm{XCS}$ and Planck cluster catalogues.

\section{Introduction}

Clusters of galaxies are the largest gravitationally collapsed structures in the Universe. While detailed studies of individual galaxy clusters are essential for obtaining an insight into the small-scale processes that influence the evolution of their baryonic components, their use as cosmological probes, and as a window to the physical processes involved in the formation and evolution of large-scale structure, requires the study of the cluster population as a whole. With this objective in mind, the XMM Cluster Survey (XCS) collaboration is carrying out a systematic search for serendipitous detections of galaxy clusters in the outskirts

\footnotetext{
^ Corresponding author: viana@ astro.up.pt
}

of publicly-available data in the XMM archive, using the $\mathrm{X}$-ray extended emission typical of galaxy clusters (Romer et al. 2001). The main scientific goals of the XCS are to (i) constrain cosmological parameters, in particular through the evolution of the cluster mass function with redshift, (ii) determine how the properties of the cluster intergalactic medium change with time, namely through the evolution of the cluster X-ray luminosity-temperature scaling relation, (iii) study galaxy evolution in clusters to high redshift.

Some of the XCS highlights are the detection, and subsequent multi-wavelength follow-up, of a $z=1.46$ cluster (XMMXCS J2215.9-1738; Stanford et al. 2006; Hilton et al. 2007, 2009, 2010), analyses of galaxy evolution in highredshift clusters (Collins et al. 2009; Stott et al. 2010), the study of the interplay between the brightest cluster galaxy 
and the intracluster medium via AGN feedback (Stott et al. 2012), the characterization of the evolution with redshift of the cluster X-ray luminosity-temperature relation (Hilton et al. 2012), and forecasts of the usefulness of XCS for the estimation of cosmological parameters and cluster scaling relations (Sahlén et al. 2009).

\section{The XCS first data release}

The XCS first data release (XCS-DR1) ${ }^{1}$ is described in Mehrtens et al. (2012). It consists of 503 optically confirmed, serendipitously detected, X-ray clusters. Of these clusters, 256 are new to the literature, 357 had their Xray emission detected for the first time, and 463 have a redshift estimate $(0.06<z<1.46)$, including 261 clusters with spectroscopic redshifts (the remainder have photometric redshifts). In addition, we have measured $\mathrm{X}$-ray temperatures, $T_{\mathrm{X}}$, for 401 clusters $\left(0.4<T_{\mathrm{X}}<14.7 \mathrm{keV}\right)$. Among the interesting subsamples of XCS-DR1 are: (i) 10 clusters at high redshift $(z>1.0$, including a new spectroscopically confirmed cluster at $z=1.01$ ); (ii) 66 clusters with high $T_{\mathrm{X}}(>5 \mathrm{keV})$; (iii) 130 clusters/groups with low $T_{\mathrm{X}}$ $(<2 \mathrm{keV})$; (iv) 27 clusters with measured $T_{\mathrm{X}}$ values in the Sloan Digital Sky Survey (SDSS) Stripe 82 co-add region; (v) 77 clusters with measured $T_{\mathrm{X}}$ values in the Dark Energy Survey (DES) region; (vi) 40 clusters detected with sufficient counts to permit mass measurements (under the assumption of hydrostatic equilibrium); (vii) 104 clusters that can be used for applications such as the derivation of cosmological parameters and the measurement of cluster scaling relations. All of the 503 galaxy clusters in XCSDR1 were confirmed through either detailed checks of the literature or our galaxy-zoo style program using data from SDSS-DR7, Stripe 82 co-add, and our own imaging/photoz survey (NXS: NOAO-XCS Survey). The 261 XCS-DR1 spectroscopic redshifts were measured using Keck, Gemini, NTT and WHT data, from SDSS luminous red galaxies and from the literature, while the 202 XCS-DR 1 photometric red-sequence redshifts were estimated using NXS, SDSSDR7 and Stripe 82 data.

The X-ray analysis methodology used to construct and analyze the XCS-DR1 cluster sample was presented in Lloyd-Davies et al. (2011). There, we described the data processing methodology applied to the 5776 XMM-Newton observations used to construct the XCS-DR1. A total of $3675(>4 \sigma)$ cluster candidates with $>50$ backgroundsubtracted X-ray photon counts were extracted from a total non-overlapping area suitable for cluster searching of 410 $\operatorname{deg}^{2}$ (280 $\mathrm{deg}^{2}$ of which associated with exposures in excess of $10 \mathrm{ks}$ ). Of these, 993 candidates were detected with $>300$ background-subtracted X-ray counts, and we demonstrated that useful temperature measurements can be obtained down to this count limit, in particular for systems with temperatures below $5 \mathrm{keV}$ (Lloyd-Davies et al. 2011).

\footnotetext{
1 www.xcs-home.org/datareleases
}

Among these candidates, we were able to optically confirm 255 galaxy clusters, presented in XCS-DR1 (Mehrtens et al. 2012). Further, in Lloyd-Davies et al. (2011) we described in detail the automated pipelines used to perform the spectral and surface brightness fitting for these candidates, as well as to estimate redshifts from the X-ray data alone.

The XMM-Newton public archive contains many thousands of observations suitable for conducting a serendipitous cluster survey. Such a large volume of data means we have to carry out most of the XCS in a fully automated manner - the only parts that are not automated are the mask making, optical follow-up, and quality control (ie. identification of non-cluster objects and spurious effects among the X-ray sources identified as possible clusters). While this automation presents a number of challenges, in terms of handling the variety and complexity of the archival data, it also has a number of benefits: not only has the entire data set been treated in a consistent and systematic way, but we are also able to run realistic simulations the XCS selection function. In Lloyd-Davies et al. (2011) we presented the methodology adopted for determining it, and showed that our extended source detection algorithm is robust to a range of cluster morphologies by inserting mock clusters derived from hydrodynamical simulations into real XMM-Newton images. These tests showed that the simple isothermal $\beta$ profile is sufficient to capture the essential details of the cluster population detected in the archival XMM-Newton observations.

The process of identification of the 3675 cluster candidates is still ongoing. We estimate that at least 300 (and up to 1700) galaxy clusters will be added to the 503 already in XCS-DR1 once the identification process is completed. Since the publication of XCS-DR1, improvements have been introduced in the several steps that need to be taken in order to identify galaxy clusters in the data present in the public XMM-Newton archive. Namely, the image pipeline was re-calibrated and updated to the latest version of the XMM-Newton Scientific Analysis System (SAS), and high background regions, as well as regions contaminated by flux from target clusters and non-cluster extended sources, are now more efficiently removed from those images. This will reduce the number of false cluster detections as we re-apply our source finding algorithm (XAPA) to all the exposures in the public XMM-Newton archive. Further, another (larger) wavelet scale has been added to XAPA in an attempt to cut down on false cluster detections and to allow us to analyze (nearby, very extended) XMM-Newton target clusters.

All NASA/IPAC Extragalactic Database (NED) clusters in the XMM-SDSS joint footprint have now been checked to see if they coincide with an XCS candidate, and the same will soon be done for the rest of the sky and for cluster catalogues too recent to be included in NED. This will not only enable the confirmation of new serendipitous detections (of already known clusters), but will also allow us to build a database of XCS-Ancillary target clusters with hundreds of entries, which will be useful for a variety of 

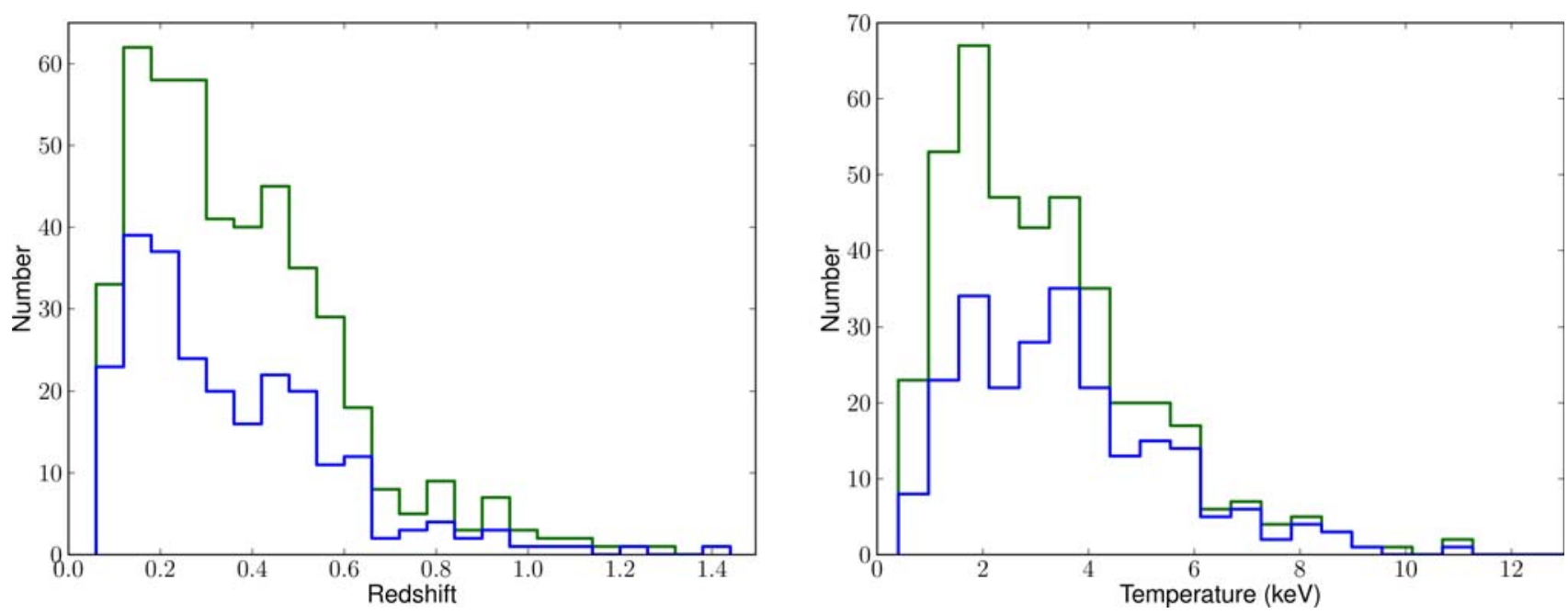

Fig. 1 The redshift distribution for the 463 clusters with measured redshifts (left), and the X-ray temperature distribution for the 401 clusters with estimated X-ray temperatures (right), in XCS-DR1 are represented by the green lines. The blue lines represent the 255 clusters in XCS-DR1 which were detected with more than 300 background-subtracted X-ray counts.

science studies where knowledge of the cluster selection function is not a critical issue, e.g. measurements of halo occupation numbers (ongoing - using galaxies in BOSS: Baryon Oscillation Spectroscopic Survey) and optical to $\mathrm{X}$-ray correlations (ongoing). We will soon be extending our XCS-Zoo identification program using additional imaging data from the Canada-France-Hawaii Telescope Legacy Survey (CFHTLS), Red Sequence Cluster Survey (RCS) and VISTA Hemisphere Survey (VHS), and later from DES.

A new non-parametric method to estimate photometric redshifts has been developed (based on measuring galaxy redshifts rather than red sequence redshifts), and will soon be applied to XCS cluster candidates in the SDSS DR8 footprint. There are ongoing spectroscopic redshift campaigns using multi-object spectroscopy at the Gemini and Magellan observatories, and eventually DES data will be used to estimate photometric redshifts, which will enable the measurement of X-ray temperatures for at least 200 XCS galaxy clusters.

The XCS selection function will be re-calculated as a function of cluster observables, like flux, temperature, redshift and angular size, for each exposure in the public XMM-Newton archive (rather than for a typical XMMNewton exposure).

\section{Applications of XCS-DR1}

\subsection{Fossil systems}

In Harrison et al. (2012) results are presented of a search for fossil systems (Ponman et al. 1994; Jones et al. 2003) that appear both in the XCS-DR1 and in the SDSS, as well as of a study of the stellar mass assembly and stellar populations of their fossil galaxies. In total, 17 groups and clusters were identified at $z<0.25$ with large magnitude gaps between the first and fourth brightest galaxies, with the total and fractional luminosity of the brightest galaxy positively correlated with the magnitude gap. All the information relevant for the classification of these systems as fossils is provided in Harrison et al. (2012). We found that the fossil systems seem to follow the same optical to X-ray scaling relations of the general population of galaxy groups and clusters, and that the brightest galaxies in fossil systems (called fossil galaxies) have stellar populations and star-formation histories which are similar to normal brightest cluster galaxies. However, at fixed group/cluster mass, the stellar masses of the fossil galaxies are larger compared to normal brightest cluster galaxies, a fact that holds true over a wide range of group/cluster masses. Further, the fossil galaxies studied also seem to be clearly more massive (by about $60 \%$, for fossil systems with $T_{\mathrm{X}} \sim 3 \mathrm{keV}$ ) than the typical Brightest Cluster Galaxy (BCG) at $z \sim 1$. This suggests that highredshift BCGs are not the progenitors of fossil galaxies, unless significant major merging events have yet to occur in BCGs. Finally, the fossil galaxies were found to contain a significant fraction of the total optical luminosity of the group/cluster, as much as $85 \%$, compared to the non-fossils, which can have as little as $10 \%$. Our results support the hypothesis that fossil systems formed early and in the highest density regions of the Universe and that fossil galaxies represent the end products of galaxy mergers in groups and clusters.

\subsection{XCS-Planck overlap}

In Viana et al. (2012) we identified 15 clusters of galaxies in XCS-DR1 that have a high probability of detection by the Planck satellite. Three of them already appear in the Planck Early Sunyaev-Zel'dovich (ESZ) catalogue (Planck Collaboration 2011). The estimation of the Planck detection probability assumes the flat Lambda cold dark matter ( $\Lambda \mathrm{CDM})$ cosmology most compatible with the 7-year Wilkinson Mi- 
crowave Anisotropy Probe (WMAP7) data (Komatsu et al. 2011). It takes into account the XCS selection function and Planck sensitivity, as well as the covariance of the cluster $\mathrm{X}$-ray luminosity, temperature and integrated Comptonization parameter, as a function of cluster mass and redshift, determined by the Millennium gas simulations (Stanek et al. 2010). We also characterized the properties of the galaxy clusters in the final data release of the XCS that we expect Planck will have detected by the end of its extended mission. We found that at $z \sim 0.1$, almost $90 \%$ of the galaxy clusters expected to be detected by Planck and which happen to be in the field of view of a XMM-Newton target observation, will also be detected by XCS (with more than 50 photon counts), slowly decreasing to slightly more than $70 \%$ at $z \sim 1$ (due to the existence of observations with low exposure times in the XMM-Newton archive). These galaxy clusters should provide valuable insights into the cross-correlations of cluster X-ray and Sunyaev-Zel'dovich properties, given our knowledge of the selection functions of both surveys. Further, the XCS will not only be able to help better characterize the sensitivity of the Planck Cluster Survey, which is essential in order to recover the scaling relations pertaining to the underlying galaxy cluster population, but will also enable Planck to recover some information on many of the galaxy clusters that are just below its detection threshold in the sky area that is common to both surveys, as well as significantly improve the estimates of the integrated Comptonization parameter for all clusters detected by Planck by aiding in the determination of cluster size.

\section{Summary}

The first data release of the XMM Cluster Survey (XCSDR1) contains 503 optically confirmed groups and clusters, among which 256 are new to the literature. They are part of 3675 cluster candidates found within a total non-overlapping sky area suitable for serendipitous cluster searching of $410 \mathrm{deg}^{2}$. The process of identification of these cluster candidates is still ongoing, and we estimate that a very conservative minimum of 300 galaxy clusters are waiting to be found among them. We expect the final sky area, suitable for cluster searching, that will be explored by XCS will be at least $600 \mathrm{deg}^{2}$, suggesting that the final XCS catalogue will contain a number of entries easily exceeding one thousand. Since the publication of XCS-DR1, several improvements have been made in our X-ray analysis pipeline. This will lead to a candidate cluster list less contaminated by spurious effects and non-cluster X-ray sources, which, together with access to CFHTLS, RCS, VHS and DES data, will speed up the confirmation process of the candidate clusters. We are also building a database of clusters that have been the target of XMM-Newton observations, which we have called XCS-Ancillary clusters, with hundreds of entries, for which there is on average much better X-ray data than for the serendipitously detected clusters in the XCS catalogue, though on the downside it is more difficult to estimate own representative they are of the overall cluster population due to a lack of selection function. The XCS data has already been used for a variety of studies, of which we highlighted here the recent characterization of fossil systems in SDSS data (Harrison et al. 2012) and of the current and expected overlap between XCS and the Planck Cluster Catalogue (Viana et al. 2012).

Acknowledgements. This work has been made possible by the ESA XMM-Newton mission, and we thank everyone who was involved in making that mission such a success. Financial support for this project includes: The Science and Technology Facilities Council (STFC) through grants ST/F002858/1 and/or ST/I000976/1 (for AKR, NM, ARL and MS), ST/H002391/1 and PP/E001149/1 (for CAC), and through a studentship (for NM). The Leverhulme Trust (for MH). FP7-PEOPLE- 2007-4-3-IRG n 20218 (for BH). Fundação para a Ciência e a Tecnologia through the project PTDC/CTE- AST/64711/2006 (for PTPV). The Swedish Research Council (VR) through the Oskar Klein Centre for Cosmoparticle Physics (for MS). ARL was supported by a Royal SocietyWolfson Research Merit Award.

\section{References}

Collins, C. A., Stott, J. P., Hilton, M., et al. 2009, Nat, 458, 603 Harrison, C. D., Miller, C. J., Richards, J. W., et al. 2012, ApJ, 752 , id. 12

Hilton, M., Collins, C. A., Stanford, S. A., et al. 2007, ApJ, 670, 1000

Hilton, M., Stanford, S. A., Stott, J. P., et al. 2009, ApJ, 697, 436

Hilton, M., Lloyd-Davies, E. J., Stanford, S. A., et al. 2010, ApJ, 718,133

Hilton, M., Romer, A. K., , Kay, S. T., et al. 2012, MNRAS, 424, 2086

Jones, L. R., Ponman, T. J., Horton, A., et al. 2003, MNRAS, 343, 627

Komatsu, E., Smith, K. M., Dunkley, J., et al. 2011, ApJS, 192, 18 Lloyd-Davies, E. J., Romer, A. K., Mehrtens, N., et al. 2011, MNRAS, 418,14

Mehrtens, N., Romer, A. K., Hilton, M., et al. 2012, MNRAS, 423, 1024

Planck Collaboration 2011, A\&A, 536, A8

Ponman, T. J., Allan, D. J., Jones, L. R., et al. 1994, Nat, 369, 462

Romer, A. K., Viana, P. T. P., Liddle, A. R., \& Mann, R. G. 2001, ApJ, 547, 594

Sahlén, M., Viana, P. T. P., Liddle, A. R., et al. 2009, MNRAS, 397, 577

Stanford, S. A., Romer, A. K., Sabirli, K., et al. 2006, ApJ, 646, L13

Stanek, R., Rasia, E., Evrard, A. E., Pearce, F., \& Gazzola, L. 2010, ApJ, 715, 1508

Stott, J. P., Collins, C. A., Sahlén, M., et al. 2010, ApJ, 718, 23

Stott, J. P., Hickox, R. C., Edge, A. C., et al. 2012, MNRAS, 422, 2213

Viana, P. T. P., da Silva, A., Ramos, E. P. R. G., et al. 2012, MNRAS, 422, 1007 